\title{
Introducing the new Protocol Exchange site
}

The Protocol Exchange (our open and free protocol repository) has a new website that allows authors to upload their protocols more easily and update them via versioning. There is also new functionality for readers, who can highlight and annotate specific parts of the text, view reader metrics (citations, views and comments) and discover similar protocols in the 'More from Protocol Exchange' section.

T

he key features of the Protocol Exchange remain the same. Protocols are not peer-reviewed or edited, although they are screened for non-scientific content and material that might pose health or biosecurity risks. As the content is not peer-reviewed or vetted, the Exchange functions as a protocol preprint server, and sharing a protocol on the Exchange should not preclude its publication in a journal. In fact, editors at Springer Nature actively encourage authors to deposit their protocols into the Exchange to improve the reproducibility of the research that we publish.

Every protocol uploaded to the Exchange is assigned a digital object identifier (DOI), which can be cited in research papers. When sharing a protocol, authors can identify any research papers (either published or unpublished) in which the protocol has been used, so the protocol can be linked to those papers when published. For unpublished papers, we can also coordinate publication of the research paper and its associated Exchange protocol so that they are published on the same day and link to one another. This means that the methods section of your research paper never needs to be outdated again-when you optimize the methodology, you can simply update your Exchange protocol by publishing a new version.

Although they are both managed by Nature Protocols editors, the Protocol Exchange and Nature Protocols are autonomous entities within Springer Nature. The Exchange is an open resource where any protocol can be freely shared, whereas Nature Protocols is a peerreviewed journal of published protocols that have been carefully selected or commissioned by its editors. The aim of Nature Protocols is to provide readers with gold-standard procedures that have been proven to work and have undergone rigorous quality control, but there are countless other protocols being used by the scientific community, which is why we set up the Exchange back in 2006 (then called the Protocols Network). Since then, we have been pleased to see the emergence of more protocol repositories, such as Bio-protocol and protocols.io, and we hope that having a choice of platforms will increase enthusiasm for protocol sharing.

At Springer Nature, the Exchange has an important role in our mission to publish open and reproducible science, and one of the most exciting aspects of this migration is that it gives us renewed impetus to promote its use among our authors. For the past decade, most of the Nature Research journals have been encouraging use of the Exchange, and a few journals (Nature, Nature Methods and Nature Cell Biology) have insisted upon deposition for specific fields in which detailed protocols are particularly important (such as stem cell research). More of the Springer Nature journals will now be encouraging deposition, and we will consider whether to promote use of the Exchange more proactively in certain areas.

We hope that, as both authors and readers, you will enjoy using the new Exchange site. Over the coming months, we aim to continue developing the site by adding features such as private sharing of protocols before publication (to assist with collaborative efforts to develop protocols) and allowing bookmarking of favorite protocols. If you have suggestions for new features or functionality, please do get in touch!

Published online: 19 June 2019

https://oi.org/10.1038/s41596-019-0199-6 